\title{
Simulation and optimization of water-wall tube panels design for power boilers
}

\author{
Maxim P. Kurepin ${ }^{1}$, and Mikhail Yu. Serbinovskiy, ${ }^{1, *}$ \\ ${ }^{1}$ PJSC “The Taganrog Boiler-Making Works “Krasny Kotelshchik”, 220 Lenina St., Taganrog, \\ 347928, Russia.
}

\begin{abstract}
The article presents a water-wall tube panels simulation technique that uses finite element method as well as the power boiler water-wall structure design optimization method. Results of the proposed method verification and its application to the design of boilers of different capacities are discussed. The technique is to replace water-wall tube panels with equivalent plates. Replacement plates' parameters calculation is reported, panels one-way heating metering is described. Optimization of the water-wall structure design is carried out in two stages: at the first stage, water-wall tube panels are replaced with orthotropic plates; at the second stage, sub-simulation with solid models is used to identify problem areas of the design. Solid-state models of the problem areas get connected to the orthotropic plates at calculated boundary conditions. The described approach improves accuracy of the simulation results and significantly reduces the models complexity and its computation time. The proposed simulation and optimization method has been tested and successfully applied to design power steam boilers with steam generating capacity of 500, 1030 and 1900 tons of steam per hour. As a result, while maintaining the specified reliability and durability, significant reduction of metal content in boiler structure is achieved.
\end{abstract}

\section{Introduction}

Modern power boiler is a sophisticated unit based on the system of membrane tube water walls gastightly connected into a water wall structure normally including a furnace, one or two connecting gas ducts and one or two convection shafts. Inside the furnace, the fuel is practically completely burnt; thermal energy form it is transferred to the heat carriers of water and steam in the water wall tubes, and to the heat exchange elements such as platens, superheaters and economizers which are placed inside the water-wall structure. All heattransfer parts with their suspender-support systems interact with the water wall structure via the sealing elements. Besides, the water-wall structure itself is suspended from and/or supported by the steel structure; it is equipped with buckstays and serves for supporting or suspending the burner devices, piping and other boiler structure parts. Thus, the water-wall structure, including all structural elements connected thereto, represents a rather sophisticated mechanical system.

\footnotetext{
*Corresponding author: Serbinovskiy_MY@tkz.power-m.ru
} 
It should be noted that thermal and hydraulic schemes of the modern power boilers and their calculation methods are almost perfect; therefore, possibilities to considerably increase boiler competitiveness are quite slim. On the contrary, there has not been given enough attention to the boilers steel structure improvement so far, although there is considerable potential in this field in terms of reducing the boilers metal consumption ensuring their reliability and longevity, which will improve specific technical and economic characteristics of power boilers. The key part in the boiler structure optimization is given to the three-dimensional numerical finite element simulation. Therefore, efficient finiteelement simulation methods development is one of the major tasks to ensure strain-stress state analysis and optimization of all-welded gastight structure design of power boilers at minimum labour input and minimum computation time. The latter is due to the use of reduced plates instead of solid water wall models that considerably decreases the model "weight” maintaining simulation accuracy.

In 1970-1980s, numerical simulations were performed mostly by approximate method of membrane water wall reduction. For example, Goldberg [1] presented a procedure for constant elastic orthotropic plates calculation without considering the difference in the water wall behavior at membrane and momental loading, which was justified by insufficient development of the simulation techniques at those times. However, such approach yielded results within an inacceptable error limit. Modern methods of water wall reduction simulation take into account heterogeneous panel behavior. An interesting investigation into gastight water walls reduction to orthotropic plates was conducted by Josip Sertić et al. [2, 3, 4]. It described analytic dependences for elastic constants of reduced plates. Nevertheless, drawbacks of this method include presentation of water wall tubes as ideally rigid bodies and neglect of potential shear energy, which over-simplifies the simulation. One more approach to membrane water wall replacement with equivalent plates offered by Hasan Nagiar et al. [5] is connected with the use of special finite elements of two different thicknesses for the membrane and bending strain components. The main weakness of this method is the neglect of shear component in the strain-stress state and determination of the orthotropic plate characteristics by purely numerical methods, which leads to additional errors.

Thus, it is necessary to develop a different method to simulate a more exact model for membrane water walls replacement with orthotropic plates by setting a stricter task to complete.

This article describes a new technique that combines analytical and numerical methods of reduced orthotropic plate elastic coefficients determination by making corrections in the elastic coefficients calculated analytically on the basis of the numerical modelling results and mandatory consideration for the shear components which essentially influence strainstress state of water walls, especially under normal water wall loads.

\section{Steam Boiler Membrane Wall as Structurally Orthotropic Plate}

Steam power boiler membrane wall is represented by the equivalent orthotropic plate, for which:

$$
\sigma=D \cdot \varepsilon-\Delta t \cdot D^{*}
$$

where $\sigma-$ is force vector, $\mathrm{D}-$ is elasticity matrix, $\varepsilon-$ is deformation vector, $\Delta \mathrm{t}-$ is variation of temperature, $\mathrm{D}^{*}$ - is temperature stress vector.

Equation (1) represents a system of six equations combining membrane and moment loads with water wall deformations: 


$$
\left[\begin{array}{c}
N_{1} \\
N_{2} \\
N_{12} \\
M_{1} \\
M_{2} \\
M_{12}
\end{array}\right]=\left[\begin{array}{cccccc}
A_{11} & A_{12} & 0 & 0 & 0 & 0 \\
& A_{22} & 0 & 0 & 0 & 0 \\
& & A_{33} & 0 & 0 & 0 \\
& & & D_{11} & D_{12} & 0 \\
\text { sym. } & & & & D_{22} & 0 \\
& & & & & D_{33}
\end{array}\right] \cdot\left[\begin{array}{c}
\varepsilon_{1} \\
\varepsilon_{2} \\
\varepsilon_{12} \\
k_{1} \\
k_{2} \\
2 k_{12}
\end{array}\right]-\left(t-t^{0}\right) \cdot\left\{\begin{array}{c}
M^{t}{ }_{1} \\
M^{t}{ }_{2} \\
M^{t}{ }_{12} \\
B^{t}{ }_{1} \\
B^{t}{ }_{2} \\
B^{t}{ }_{12}
\end{array}\right\}
$$

where $N_{1}, N_{2}, N_{12}$ - are forces, $M_{1}, M_{2}, M_{12}$ - are moments acting in the membrane water wall, $A_{11}, A_{12}, A_{22}, A_{33}$ - are elasticity matrix members for membrane loads, $D_{11}, D_{12}, D_{22}$, $D_{33}$ - are elasticity matrix members for moment loads, $\varepsilon_{1}, \varepsilon_{2}, \varepsilon_{12}$ - are membrane deformations, $k_{1}, k_{2}, k_{12}$ - are neutral surfaces curvatures of the equivalent orthotropic plate, $t$ - is current plate temperature, $t^{0}$ - is initial plate temperature, $M^{t}{ }_{1}, M^{t}{ }_{2}, M_{12}^{t}$ - are membrane stress components caused by single temperature rise in a completely fixed model, $B_{1}^{t}, B^{t}{ }_{2}, B^{t}{ }_{12}$ - are bend stress components caused by single temperature rise in a completely fixed model.

Water wall shear stiffness influence is described as follows:

$$
\left\{\begin{array}{l}
S_{1} \\
S_{2}
\end{array}\right\}=\left[\begin{array}{ll}
E_{11} & E_{12} \\
E_{12} & E_{22}
\end{array}\right] \cdot\left\{\begin{array}{l}
\gamma_{1} \\
\gamma_{2}
\end{array}\right\}
$$

where $s_{1}, s_{2}$ - is shearing force, $E_{11}, E_{12}, E_{22}$ - are matrix members of shearing stiffness, $\gamma_{1}, \gamma_{2}$ - is shearing deformation.

Members of stiffness matrix $[A],[D],[E]$ and temperature stresses $\left\{M^{t}\right\},\left\{B^{t}\right\}$ are expressed via stiffness parameters of the membrane walls determined analytically or by numerical modeling. In the latter case, a small part of water wall shall be simulated (fig. 1, a) and determined the boundary conditions for separate loading: tension along and across the tube axes, pure bending of the water wall in two planes parallel and normal to the tube axes, pure bending with force along and across the tube axis, plane bending under transverse load; (fig. 1, b) bend in 2 planes, parallel and normal to the tube axes under normal action to the water wall (fig. 2).

a

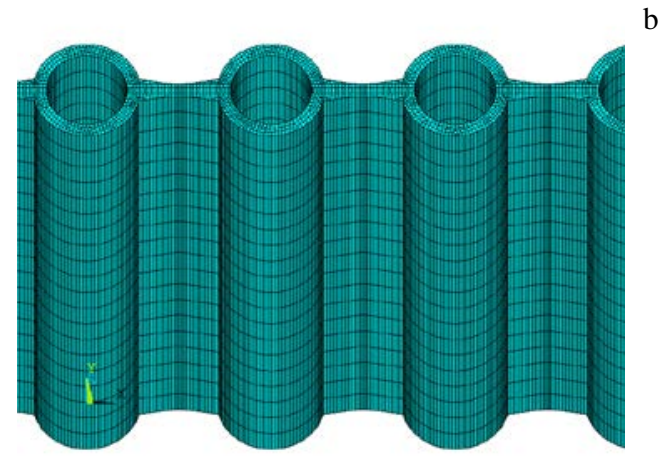

$\mathrm{b}$

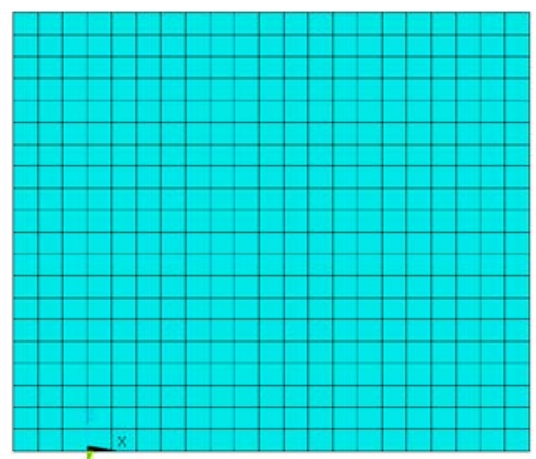

Fig. 1. Finite-element models of the membrane water wall: a) solid, b) shell.

The water wall model deformations are determined, and by these values, elastic coefficients of reduced plates are calculated [6].

To determine the equivalent plates parameters, a system of equations is formed describing loads given in fig. 2:

Tension along and across the tube axis (loading 1 and 2, fig. 2), stress components in the plate of the rectangular cross-section equal [7], for loading 1 equal the following: $\sigma_{x}=$ $\frac{F}{h l} ; \sigma_{y}=\sigma_{z}=\tau_{x y}=\tau_{x z}=\tau_{y z}=0$, for loading 2 stress components equal the following: 
$\sigma_{x}=\sigma_{y}=\tau_{x y}=\tau_{x z}=\tau_{y z}=0 ; \sigma_{z}=\frac{F}{h \cdot 2 a}$, where $F-$ is tensile strength, $H, h-$ is thickness of the orthotropic plate for the simulated membrane water wall. Then, equations in (2) are as follows:

$$
\begin{gathered}
E_{x, \mathrm{p}}=\frac{F \cdot 2 a}{d x_{1} \cdot l \cdot h}, \mu_{x y}=\frac{d y_{1} \cdot 2 a}{d x_{1} \cdot h}, \mu_{x z}=\frac{d z_{1} \cdot 2 a}{d x_{1} \cdot l}, \\
E_{z, \mathrm{p}}=\frac{F l}{d z_{2} \cdot 2 a \cdot h}, \mu_{y z}=\frac{d y_{2} \cdot E \cdot 2 a}{F},
\end{gathered}
$$

where $d x_{i}, d y_{i}, d z_{i}$ - are displacements of extreme nods of the water walls under the relevant loading obtained by numerical experiment.
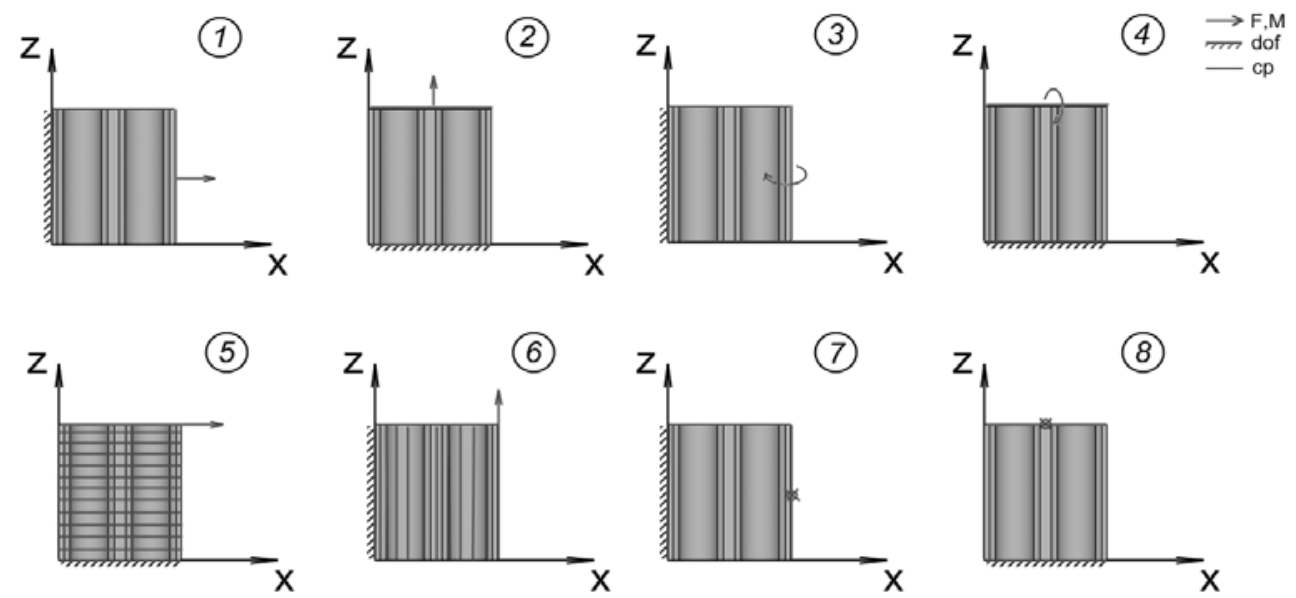

Fig. 2. Membrane water wall model loading: 1) tension across the tube axis; 2) tension along the tube axis; 3) pure bending in $X Y$ plane by moment $M_{z}$; 4) pure bending in $Y Z$ plane by moment $M_{x}$; 5) pure shearing by the force along the $O X$ axis; 6) pure shearing by the force along the $O Z$ axis; 7) bending in XOY plane under action of the shear load; 8) bending in ZOY plane under action of the shear load.

The pure bending in $X Y$ plane by moment $M_{z}$ and in $Y Z$ plane by moment $M_{X}$ (loading 3 and 4, fig. 2), stress components are equal to [7]: for loading 3: $\sigma_{x}=\frac{E_{x} \cdot y}{R_{x}} ; \sigma_{y}=\sigma_{z}=$ $\tau_{x y}=\tau_{x z}=\tau_{y z}=0$, for loading 4: $\sigma_{x}=\sigma_{y}=\tau_{x y}=\tau_{x z}=\tau_{y z}=0 ; \sigma_{z}=\frac{E_{z} \cdot y}{R_{z}}$, where $R_{z}$ and $R_{x}$ - are curvature radii after bending in planes $X Y$ and $Y Z$, respectively, equaling to: $R_{z}=\frac{E_{x} \cdot I_{z}}{M_{z}} ; R_{x}=\frac{E_{z} \cdot I_{x}}{M_{x}}$; using the equation of constant thickness plate flexures: $v=-\frac{M \cdot z^{2}}{2 \cdot E \cdot I}$, we obtain values in the extreme points of the water wall along the axis $\mathrm{y}-d y 4$, dy3 and Young's modulus in the direction of $\mathrm{OX}$ and $\mathrm{OZ}$ axes:

$$
E_{x, \text { bend }}=\frac{6 M \cdot(2 a)^{2}}{d y_{3} \cdot l \cdot h^{3}}, E_{z, \text { bend }}=\frac{6 M \cdot l^{2}}{d y_{4} \cdot 2 a \cdot h^{3}} .
$$

Assuming that $E_{z, b e n d}=E_{z, m}$, we obtain the orthotropic plate thickness $(h)$ :

$$
h=\sqrt{\frac{6 \cdot M \cdot l \cdot d z_{2}}{F \cdot d y_{4}}},
$$

and values of the other plate parameters. It should be mentioned that the Young's modulus values obtained at the model tests for bending and tension in the direction across the tube 
axis differ substantially. Small bending stiffness of the water wall results in small bending stiffness of the water wall fins compared to the tube bending stiffness. Fulfillment of the condition $E_{z, \text { bend }}=E_{z, m}$ is possible provided that only additional horizontal tension rods are used. Therefore, additionally, the plate stiffness in the direction of $O X$ axis equal to the value obtained at the bending test is applied. Then, along the tube axis rods with the specified pitch are installed, perpendicular to the tubes, compensating the lack of stiffness at tension, whose rods' cross section $f_{s}$ is defined by the resistance force equation of the water wall $F_{x, W W}$ and the sum of forces of the plate $F_{x, \text { plate }}$ and rods $F_{x, \text { rod }}$ :

$$
\begin{gathered}
F_{x, W W}=F_{x, \text { plate }}+2 \cdot F_{x, \text { rod }} \\
E_{x, m} \cdot l \cdot h=E_{x, \text { bend }} \cdot l \cdot h+2 \cdot E \cdot f_{s} \\
f_{s}=\frac{\left(E_{x, m}-E_{x, \text { bend }}\right) \cdot l \cdot h}{2 \cdot E}
\end{gathered}
$$

The cross-section of the compensating rods depends on the water wall section parameter $l$, for the actual water wall, the model accuracy depends on the rod pitch. When simulating the boiler membrane water wall structure, we recommend choosing 100-300 mm pitch between the rods.

The pure shear by forces along the extreme water wall planes by the $O X$ and $O Z$ axes (loading 5 and 6, fig. 2), stress components are equal to [7]: for loading 5: $\sigma_{x}=\sigma_{y}=\sigma_{z}=$ $\tau_{x y}=\tau_{y z}=0 ; \tau_{z x}=\frac{F}{2 a \cdot h} ;$ for loading 6: $\sigma_{x}=\sigma_{y}=\sigma_{z}=\tau_{x y}=\tau_{y z}=0 ; \tau_{x z}=\frac{F}{l \cdot h}$; having defined the displacement of the plane extreme points as $d x_{5}$ и $d z_{6}$, using (2), considering minor shear angle and constraint equation application for the bend compensation in planes parallel to the planes of the applied forces, we obtain the following for XOZ plane:

$$
G_{x z}=\frac{F \cdot l}{2 a \cdot h \cdot d x_{5}}, G_{z x}=\frac{F \cdot 2 a}{l \cdot h \cdot d z_{6}} .
$$

The obtained shear $G_{x z}$ module is not equal to $G_{z x}$ module, their relative difference is about $40 \%$ for the water wall section with $l=2 a$. However, for stress condition of the actual structure, the influence on the shear component is minor; therefore, this error can be ignored. We recommend that $G_{x z}$ values for the system (2) are assumed by the greater of the two shear module values. For the membrane water walls, in all cases, the calculated $G_{x z}$ $>G_{z x}$.

The bend of the water wall section under the shear load action (loading 7 and 8, fig. 2), stress components are equal to [7]: for loading 7: $\sigma_{y}=\sigma_{z}=\tau_{y z}=0 ; \sigma_{x}=-\frac{F \cdot(l-x) \cdot y}{I_{z}}$; $\tau_{x y}=g_{1}(x, y) ; \tau_{y z}=g_{2}(x, y)$; for loading $8: \sigma_{x}=\sigma_{y}=\tau_{x y}=0 ; \sigma_{z}=-\frac{F \cdot(l-z) \cdot y}{I_{x}} ; \sigma_{x}=$ $\sigma_{y}=\tau_{x y}=0 ; \sigma_{z}=-\frac{F \cdot(l-z) \cdot y}{I_{x}} ; \tau_{x z}=f_{1}(x, y) ; \tau_{y z}=f_{2}(x, y)$.

Using the results of simulation and the equation of crane deflection as a girder of rectangular cross-section under shear force [7] of:

$$
v=v_{\text {bend }}+v_{\text {shear }}=\frac{F \cdot l^{3}}{3 \cdot E \cdot I}+k \frac{F \cdot l}{G \cdot S}
$$

where $v_{\text {bend }}$ - is the deflection due to bend, $v_{\text {shear }}$ - is the deflection due to shear, $k-$ is uneven shearing stress distribution coefficient under the transverse bend depending on the cross-section form and computed, for example, using numerical model, we obtain the following: 


$$
G_{x y}=\frac{F \cdot 2 a}{l \cdot h \cdot\left(d y_{7}-\frac{4 \cdot F \cdot(2 a)^{3}}{E_{x} \cdot l \cdot h^{3}}\right)} ; G_{y z}=\frac{F \cdot l}{2 a \cdot h \cdot\left(d y_{8}-\frac{4 \cdot F \cdot l^{3}}{E_{z} \cdot 2 a \cdot h^{3}}\right)} .
$$

It should be mentioned that at some water wall parameters these formulas can give incorrect values of shear modules. In this case, the following order of defining $G_{x y}$ and $G_{y z}$ is recommended : 1) the target value of shear module shall be taken equal to $10^{6}-10^{8}$ MPa, calculation with loading (7) or (8) shall be made and $v_{\text {bend, } i}^{*}$ shall be defined which is the bend deflection regardless the shear; 2) the shear deflection value $v_{\text {shear }, i}^{*}$ shall be using formula $v_{\text {shear }, i}^{*}=d y_{i}-v_{\text {bend, } i}^{*}$, where $d y_{i}$ - is deflection value of the extreme middle points of the water wall section in the numerical experiments under loading (7) and (8); 3) the missing shear modules shall be defined with the following formulas:

$$
G_{x y}=\frac{F \cdot 2 a}{l \cdot h \cdot v_{\text {shear }, 7}^{*}}, G_{y z}=\frac{F \cdot l}{2 a \cdot h \cdot v_{\text {shear }, 8}^{*}} .
$$

Weight loads take into account the reduced density of the plate material equaling to:

$$
\rho=\frac{\rho_{m} \cdot s_{a}}{a \cdot h},
$$

where $\rho_{m}$ - is metal density of the membrane water wall, steel density, $s_{a}-$ is cross-section area of $a$-wide membrane water wall.

Special programs for elastic coefficients numerical determination for reduced plates with compensating rods have been created and used successfully, for example, «Ortek» copyrighted program verified on the basis of experimental data [1]. Three deformations of finned tubes water walls of the following dimensions (in $\mathrm{mm}$ ) are simulated: tube diameters are $-42 \mathrm{~mm}, 32 \mathrm{~mm}$ and $32 \mathrm{~mm}$; tube wall thickness are $-5.1 \mathrm{~mm}, 6.0 \mathrm{~mm}$ and $4.1 \mathrm{~mm}$; tube pitch is $-72 \mathrm{~mm}, 47 \mathrm{~mm}$ and $71.5 \mathrm{~mm}$; fin root thickness is $-10 \mathrm{~mm}, 8 \mathrm{~mm}$ and 9 $\mathrm{mm}$; fin top thickness $-5.0 \mathrm{~mm}, 4.6 \mathrm{~mm}$ and $3.3 \mathrm{~mm}$, respectively. Width of water wall sections for which the cross section deflection angles are determined conform to 7, 9 and 7 tube pitches, i.e. $35.7 \mathrm{~mm}, 54.0 \mathrm{~mm}$ and $28.7 \mathrm{~mm}$. The water wall length in the experiments along the tubes is $500 \mathrm{~mm}$. The water wall material low-alloy steel (chemical composition, \%: C 0.08-0.15, Cr 0.9-1.2, Mo 0.25-0.35, V 0.15-0.30), Young's modulus is $E=20900$ MPa. The deflection angle from the unit moment of the cross sections conforming to the fin tops (fin top edges from the unit moment) for the solid water wall model are 6.82e-6, 4.36e6 and 1.79e-5 rad/H respectively; for the water wall model based on the orthotropic plates they are $6.85 \mathrm{e}-6,5.06 \mathrm{e}-6$ and $1.78 \mathrm{e}-5 \mathrm{ad} / \mathrm{H}$ respectively; analytical angles as per [1] are 6.86e-6, 4.58e-6 and 1.75e-5 rad/H. Experimental values of angles are 7.44e-6, 4.48e-6 and $1.61 \mathrm{e}-5 \mathrm{rad} / \mathrm{H}$. Respectively, the simulation errors for the solid water wall model are -8.3 , 2.6 and $10.9 \%$; for the shell water wall model are $-7.9,12.8$ and $-10.2 \%$, and when comparing to the theoretical values, they are $-0.6,4.8$ and $-1.8 \%$ for the solid model and $0.2,-10.3,-1.2 \%$ for the shell model.

Difference between the simulation results using the methods described and physical experiment results is \pm 10 - $13 \%$. Similar results difference values are obtained for physical experiments and analytically as per [1]. It should be noted that the deflection angle value of the cross sections is sensitive to geometrical characteristics of the fin tube; therefore, it is not possible to obtain more exact results in this case as tolerances for the geometrical dimensions of the fin tubes give a wider spread in values of the target deflection angle. The difference between the simulation results and the results obtained analytically as per [1] is within $\pm 5 \%$ for the solid models and \pm 10 - $11 \%$ for the shell models. 
Therefore, the shell model of the membrane water wall based on the orthotropic plate with mechanical properties calculated using the proposed method gives great results which are in line with analytical calculations and with calculations for solid models.

The efficiency of the proposed method with optimization of the water wall structures increases when used along with solid simulations, then, at the first stage, optimum geometrical models are created with the use of equivalent orthotropic plates as well as calculation and analysis of the results, including problematic structure areas identification; and at the second stage, sub-simulation of the problematic areas are performed [6]. Such approach allows considerable reduction in the labour intensity for simulation models creation and for calculation time with the simultaneous increase in accuracy of the calculation results for all nods of the sophisticated models.

\section{One-Sided Heating}

It is known that, when simulating one-sided heating of all-welded water wall panels, it is necessary to take into account the fact that the radiant heat flux is taken up by the panel surface depending on the intensity of illumination of each point. In order to determine the water wall temperature by means of the analytical calculations, values of the taken up heat flux are used, however, such approach is labour-intensive and difficult to implement in practical design. Therefore, the authors have developed a new simulation method for temperature field and strainstress state of water walls using finite element method, in particular, with the use of ANSYS software [8].

For simulating the temperature field using FEM, a task is to have a heat flux boundary condition in each boundary nod; therefore, it is necessary to calculate the angular factors for the radiant heat flux. The angular luminosity factors are calculated analytically using the dependence [8]. As a result, temperature fields and temperature strain are obtained (see fig. 3).

One-sided heating is normally considered at the second stage of the boiler calculation; when shell models based on the orthotropic plates are changed for solid models of the special boiler places in simulation. However, temperature factors can be taken into account yet at the stage of "large" shell model of water wall structure by filling the temperature matrices $\left[M^{t}\right]$ and $\left[B^{t}\right]$. At that, thermos-elastic moments are preliminarily determined, for example, as per $[1,8]$.

The boiler unit simulations, using the abovementioned methods, allow revealing the bottlenecks and optimizing the designs of the furnace bottom ash hoppers of the oncethrough pulverized steam boiler with steam output of 1900 tons of steam per hour. Furnace bottom ash hopper truss buckstays, buckstays and water wall and buckstay fasteners, and their arrangement on the water walls are optimized. This results in the metal economy of more than 100 tons.

As a result of boiler furnace and convection shaft water walls simulation, a number of solutions are proposed, which allow ensuring target reliability and durability, while considerably reducing the costs compared to the traditional solutions. Similar results are obtained for drum gastight boilers with steam output of 500 tons of steam per hour, where the metal consumption of the furnace bottom ash hopper is reduced by $6-20$ tons, and metal consumption of the suspender-support system of the boiler water wall structure is also considerably reduced. 
a
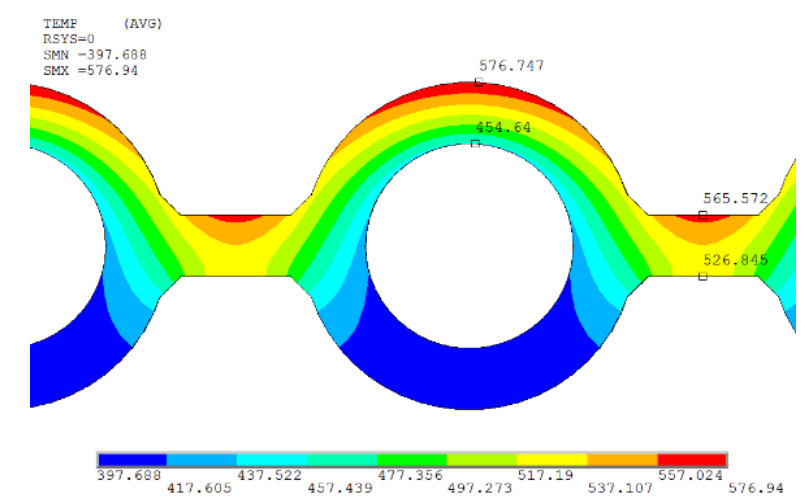

b

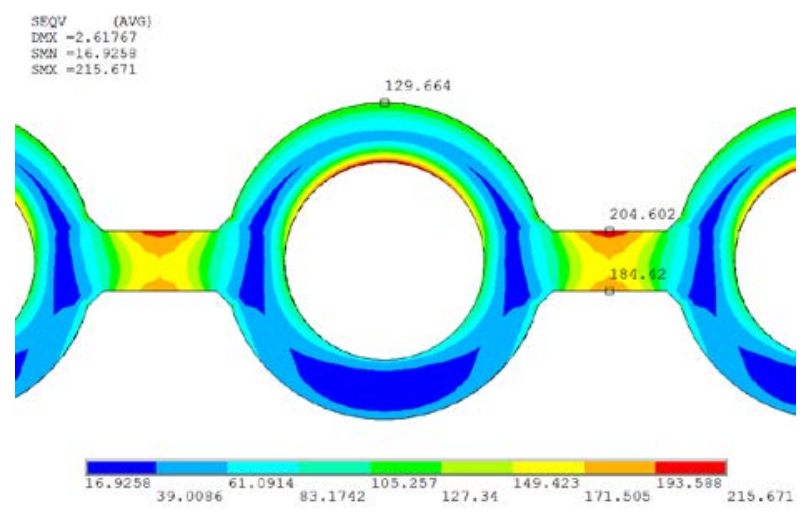

Fig. 3. Simulation results of the one-sided heating of the free panel as per FEM: a - temperature field, ${ }^{\circ} \mathrm{C}$; b - strain field, MPa.

To sum up, furnace simulation with helically wound tubes for once-through boiler with steam output of 1030 tons of steam per hour is performed. The simulation allows choosing and optimizing boiler water wall support structure system in all its parts: furnace bottom ash hoppers, lower radiation part of the furnace with helically wound tubes, furnace upper radiation part with vertical tubes, and boiler transient gas duct and convection shaft.

Numerical calculation of water wall structure allows obtaining specified force distribution across the boiler hangers or reactions in the supports for the self-supporting boilers, solving a variety of accompanying tasks of design optimization for gastight water wall supporting parts, buckstay fastening system, burner hangers, insulation selection, etc. (see fig. 4).

\section{Conclusions}

1. An effective method of membrane screens simulation and all-welded gas-tight boiler screen boxes simulation using equivalent orthotropic plates is proposed. The method includes a new technique to form equivalent plates with additional tension rods and calculates geometric and deformation parameters of these plates. The technique allows simulating the stress-strain state of the screens with high accuracy.

2. An effective method of optimizing boiler structures with all-welded gas-tight screens is proposed, combining in one and the same model equivalent orthotropic plates that replace screens and solid-state models of screen parts. The model provides desired result combines with low optimization complexity. 


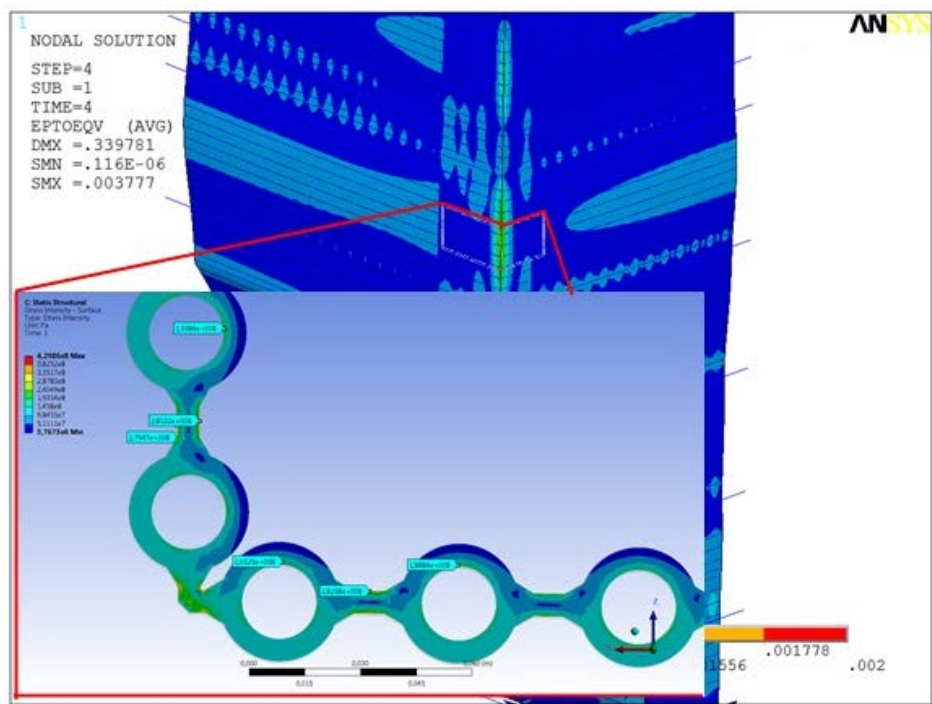

Fig. 4. Examples of sub-simulation modeling for strain-state determination of boiler furnace all-welded gastight water walls.

3. An original simulation technique for one-sided screens heating, temperature field and stress-strain state of screens using the finite element method is described.

4. The effectiveness of the proposed methods is confirmed by verification on the basis of experimental data and the use of real energy boilers design, and has proved the following:

- ability to simulate the stress-strain state of boiler structures on medium-sized personal computers;

- $\quad$ significant reduction in complexity of created models and in computational time;

- possibility of significant reduction in metal consumption (material consumption) for optimized boiler designs with steam output from 100 to 1900 tons of steam per hour.

Thus, the method described results in optimization of the water-wall structure design while maintaining the specified reliability and durability of the boiler and offering obvious economic benefits of optimization.

\section{References}

1. A.I. Goldberg, V.S. Koryagin, S.I. Mochan, E.M. Tyntarev, Calculation and Design of All-Welded Water Walls of the Boiler Units (Power, Leningrad, 1975)

2. J. Sertić, I. Gelo, D. Kozak, D. Damjanović, P. Konjatić, Theoretical Determination of Elasticity Constants for Steam Boiler Membrane Wall as Structurally Orthotropic Plate, Tehnički vjesnik, v. 20(4), pp. 697-703 (2013)

3. J. Sertić, D. Kozak, I. Samardžić, Calculation of Reaction Forces in the Boiler Supports Using the Method of Equivalent Stiffness of Membrane Wall, The Scientific World Journal, v. 2014, Article ID 392048, http://dx.doi.org/10.1155/2014/392048

4. B. Gaćeša, V. Milošević-Mitić, T. Maneski, D. Kozak, J. Sertić, Numerical and Experimental Strength Analysis of Fire-Tube Boiler Construction, Technical Gazette, 18(2), pp. 237-242 (2011) 
5. H.M. Nagiar, T.Dj. Maneski, V.O. Milošević-Mitić, B.M. Gaćeša, N.M. Andjelić, Modeling of the Buckstay System of Membrane-Walls in Watertube Boiler Construction, Thermal Science, v. 18(1), pp. S59-S72 (2014)

6. Patent 2568783 RF MPK \# G06N7/06. Method of Design Analysis and Optimization of the Boiler with Finned Water Walls. / Kurepin M.P., Serbinovskiy M.Y., Ivanenko V.V. - Application date 24.10.2014., Application No.2014143062, Published on. 20.11.2015. Issue No.32.

7. S.P. Timoshenko, J. Gudier, Elastic Theory. 2nd Edition (Science. Office of Editor-inChief of Physics-mathematical Literature, Moscow, 1979)

8. M.P. Kurepin, M.Y. Serbinovskiy, Simulation of One-Sided Heating of Boiler Unit Membrane-Type Water Walls, Thermal Engineering, v. 64(3), pp. 209-215 (2017) 